\title{
Computer-Mediated Communication Systems
}

\author{
Bin Yu
}

yubby2009@gmail.com, Beijing Institute of Graphic Communication, Beijing, China

\begin{abstract}
The essence of communication is to exchange and share information. Computers provide a new medium to human communication. CMC system, composed of human and computers, absorbs and then extends the advantages of all former formats of communication, embracing the instant interaction of oral communication, the abstract logics of printing dissemination, and the vivid images of movie and television. It also creates a series of new communication formats, such as Hyper Text, Multimedia etc. which are the information organizing methods, and cross-space message delivering patterns. Benefiting from the continuous development of technique and mechanism, the computer-mediated communication makes the dream of transmitting information cross space and time become true, which will definitely have a great impact on our social lives.
\end{abstract}

Keywords: communication, information, computer-mediated communication

Acknowledgement: Thanks to the instruction of Professor Wang Jingshan, this paper was finished in time.

The essence of communication is to exchange and share information. In contemporary age, media of communication have various forms and states, especially the network medium. But no matter how special it is, the nature of network mediated by computers is still information's transmitting and sharing.

Computer-mediated communication is just a new form of human communication with all the former kinds of communication features included. It absorbs furthermore extends the advantages of the former formats, embracing the instant interaction of oral communication, the abstract logics of printing dissemination, and the vivid images of movie and television. Besides that, it creates a series of new communication formats, such as Hyper Text, Multimedia etc. which are the information organizing methods and cross-space message delivering patterns. Benefiting from the continuous development of technique and mechanism, computer-mediated communication makes the dream of transmitting information cross space and time come true, which will definitely have a great impact on our social lives.

\section{Definition of Computer-Mediated Communication}

Communication is a process by which people exchange information or express their thoughts and feelings. It has many formats, such as Interpersonal Communication, Organizational Communication, Oral Communication, Small Group Communication, Intercultural Communication and so on, but what we discussing here is Computer-mediated Communication, a kind of communication based on computers and networks.

Computer-mediated Communication (CMC) is defined as any communicative transaction that occurs through the use of two or more networked computers ${ }^{1}$. Popular forms of CMC include email, video, audio or text chat, bulletin boards, list-services and MMOs (massively multiplayer online game). These settings are changing rapidly with the development of new technologies.

Computer-mediated Communication is a system consisting of human and computer, which means that it is alive instead of some rigid facilities. The participation of humans makes themselves creators, users, ameliorators, furthermore the core of the computer-mediated communication system, which means that without humans, the system would only have cold apparatus left. The com-

\footnotetext{
${ }^{1}$ McQuail, Denis. (2005). Mcquail's Mass Communication Theory. 5th ed. London: SAGE Publications.
} 
bination of humans and computers, or to say networks, forms a complicated information transmitting system aiming at information exchanging and sharing freely. There are some general features of information system in the Computer-mediated Communication System, together with some special features of human social system.

\section{Components of Computer-Mediated Communication System}

Harold Dwight Lasswell's well known comment on communications is "5W"mordel, including: Who (says), What (to), Whom (in), What Channel (with) and What Effect. According to his theory, CMC system, as a new form of communication with its own characteristics, can also be divided into five components: Subject, Host, Information resource, Channel, Relations and Effects.

\subsection{Subject}

The Subject of CMC system is human, called Network Users, who can be creators, providers and recipients of the network. Therefore the subject performs roles as the initiator, the media and also the target. Since CMC system implies reciprocal features, the roles of people in the network are changeable.

Being different from other forms of communication, the subject, i.e. human has much closer connections with CMC system itself, even can be embedded part of the system. Meanwhile, human is also part of the social system which forms the border of CMC system, in consequence, human become a bridge between these two systems. Through this bridge, the role of CMC system in the social system has been broadened and deepened.

The Subject of CMC system has a great amount, also with complicated relations inside. For example, at present, China has more than 300 million internet users, who are not only users of network information service, but also service providers at the same time. Holding different purposes on communicating through $\mathrm{CMC}$ system, they have a great influence on the development and evolution of this system.

Since the relationship among the subjects of CMC system is so important that its establishment and maintenance rely on the communicating though network, furthermore, on the communicating in the real world - the social system. Here this network relationship is actually a presentation of the real one.

\subsection{Host}

In computer networking, a network host (Internet host, host, or Internet node) is a computer connected to the Internet - or more generically - to any type of data network. A network host can host information resources as well as application software for providing network services.

The host is the most important part of a CMC system. It is the main unit which constitutes a communication network node, and it is also a center of the information resources. A special host system can be either a client, or a server, both of which are the base of networks communication.

In CMC system, a host includes not only hardware, but also software, which is a dynamic integration of them. A host system carries out a communication process by using the network equipments and the network protocol- TCP/IP etc. according to the network users' concrete instruction. In turn, the host system accomplishes the developments of information quantities and services. In brief, an isolated computer without network is just a calculator or a processing unit with the ability of dealing with information. But once it joins internet, forming a CMC system, then it will undergo a qualitative change in terms of dealing and processing. This is the unique power of the whole communication system.

\subsection{Information Resource}

Network information is a summation of all messages transmitted by the computer network and memorized on the network nodes. As for the network information resource, it can be compre- 
hended as "a summation of various information resources gained by using the network. It doesn't include all the information put in the internet, but only the section which meets the information demands of users. In consequence, the scope of Network Information Resource is narrower than that of Network Information.

Network information resources are contents and objects of network communication. During the process of $\mathrm{CMC}$, the network information is executed as the commutation of meanings which is also the essence of $\mathrm{CMC}$. CMC makes the network information getting rid of the tie of concrete medium; hence CMC becomes a pure information communication, which is a great leap of human communication.

The network information resources have characteristics on huge amounts, diverse types, easy access, wide distribution and fast transmission, so it can achieve a quickly accurate information dissemination process, which makes CMC different from other forms of communication.

\subsection{Channels}

Channel of CMC means a path of network information transmitting, which puts different subject together, making the transmission of network information smooth. It is the premise and foundation of CMC. The effects of CMC are largely determined by the characteristic and state of channel.

The channel of CMC is generally made up of network facilities, network news transfer protocols (NNTP) and network media. The facilities and media are hardware, and the protocols are software of CMC channel. CMC channel includes not only the seeable material passages, such as electric cables, but also includes various software that deliver the network information, such as NNTP, so it is a dynamic integration of hardware and software.

The network media have different forms, which can be common communicating electric cables or fibers, or submarine cables, even can be a man-made satellite data communication channel. When they are in the process of CMC, network users don't pay much attention to what actually the form of channel is, because internet provides redundant paths all the time, through which network information divided into many data packages can communicate fluently, therefore no one cares about how. But under some special circumstances, the varieties of CMC channel are necessary, for example, in 2006 there was a heavy earthquake in the realm of South China Sea, which broke several submarine cables, and caused many Chinese MSN users failing in log in.

\subsection{Relations and Effects}

Generally speaking, network information resources have potential values more or less. After the process of transmitting and accepting of Network information, there are some social impacts having been produced, and that are the effects of CMC. These effects are the essential part of CMC system, and a presentation of the social functions of CMC.

The effects may be positive, are also likely to be passive. The positive ones are called Plus Effect, whereas Negative Effect. The purpose of CMC should be enlarging the Plus Effect and restraining the Negative one.

Sometimes the effects of CMC can be seen as the establishment and maintenance of various relationships among network users. In the other kinds of communication, the subjects' relationships are a kind of invisible social existence; whereas in CMC system, the relationship between network users can be reflected by interlinkages and dynamic structures, which are also the expression of the function and fragment of CMC system.

Being similar to all the other kinds of communication, CMC system has dynamic, serial and structural features. $\mathrm{CMC}$ is a reciprocal dynamic process of information communication, and it is an orderly information transmitting system, which has its stable structures. Each element of this system is important and basic to the process of communication. Being dynamic and orderly, CMC system is complicated therefore. Having stable structures makes it possible to understand and analyze the system. CMC not only has the general features of common information communication system, but also some particular characteristics of itself. 


\section{References}

Stanley, J.B. (2008). Introduction to Mass Communication: Media Literacy and Culture (4th edition). Beijing: China Renmin University Press.

McQuail, D. (2005). Mcquail's Mass Communication Theory. 5th ed. London: SAGE Publications.

Schramm, W. \& Porter, W.E. (2007). Men, Women, Messages, and Media: Understanding Human Communication (2nd edition). Beijing: Peking University Press.

Kasdorf, W.E. (2007). The Columbia Guide to Digital Publishing. Suzhou: Suzhou University Press.

\section{About the Author}

Bin Yu

Is graduate student of Beijing Institute of Graphic Communication. 